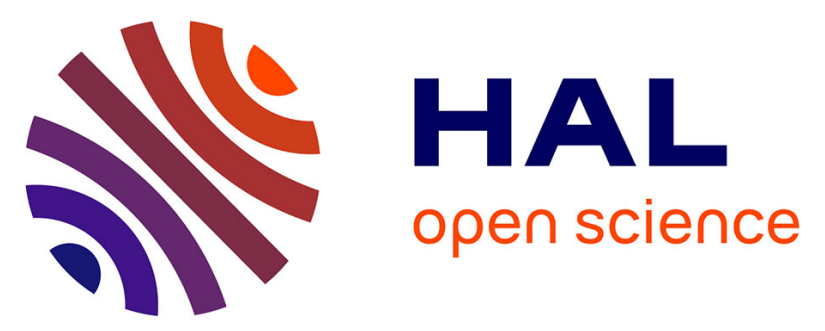

\title{
An Efficient Cross-Layer Design for Multi-hop Broadcast of Emergency Warning Messages in Vehicular Networks
}

Abir Rebei, Fouzi Boukhalfa, Haifa Touati, Mohamed Hadded, Paul Mühlethaler

\section{- To cite this version:}

Abir Rebei, Fouzi Boukhalfa, Haifa Touati, Mohamed Hadded, Paul Mühlethaler. An Efficient CrossLayer Design for Multi-hop Broadcast of Emergency Warning Messages in Vehicular Networks. AINA2021 - 35th International Conference on Advanced Information Networking and Applications, May 2021, Toronto, Canada. hal-03163181

\section{HAL Id: hal-03163181 https://hal.science/hal-03163181}

Submitted on 9 Mar 2021

HAL is a multi-disciplinary open access archive for the deposit and dissemination of scientific research documents, whether they are published or not. The documents may come from teaching and research institutions in France or abroad, or from public or private research centers.
L'archive ouverte pluridisciplinaire HAL, est destinée au dépôt et à la diffusion de documents scientifiques de niveau recherche, publiés ou non, émanant des établissements d'enseignement et de recherche français ou étrangers, des laboratoires publics ou privés. 


\section{An Efficient Cross-Layer Design for Multi-hop Broadcast of Emergency Warning Messages in Vehicular Networks}

1 Abir Rebei IResCoMath, Tunisie, abirrebei9@gmail.com

2 Fouzi Boukhalfa VEDECOM, France, fouzi.boukhalfa@vedecom.fr

3 Haifa Touati IResCoMath, Tunisie, haifa.touati@ cristal.rnu.tn

4 Mohamed Hadded VEDECOM, France, mohamed.elhadad@ vedecom.fr

5 Paul Muhlethaler INRIA, France, paul.muhlethaler@inria.fr

Summary. The main objective of Vehicular ad hoc networks (VANETs) is to make road transportation systems more intelligent in order to anticipate and avoid dangerous, potentially life-threatening situations. Due to its promising safety applications, this type of network has attracted a lot of attention in the research community. The dissemination of warning messages, such as DENMs (Decentralized Environmental Notification Messages), requirse an efficient and robust routing protocol. In previous studies, the active signaling mechanism has shown its ability to prevent collisions between users trying to allocate the same resource. In this paper, we propose an original message forwarding strategy based on the active signaling mechanism. Our proposal disseminates warning messages from a source vehicle to the rest of the network while minimizing the access delay and the number of relay nodes. For this purpose, a special time slot is dedicated to forwarding emergency warning messages. To avoid access collisions on this slot, the active signaling scheme we propose favours the selection of the furthest node as the forwarder. We carry out a number of simulations and comparisons to evaluate the performances of the scheme.

Keywords - VANETs, broadcast protocol, Cross-layer design, TDMA, Active signaling, Multi-hop communication, DENM, Network simulation.

\section{Introduction and motivation}

One of the most prominent technologies in the field of intelligent transportation systems (ITS) that has drawn significant interest from researchers and industry is Vehicular Ad-hoc Networks (VANETs). VANETS are essentially an extension of the unerlying principles of Mobile Adhoc Networks (MANETs), while featuring a number of alterations.

VANETs provide a wide range of applications covering both safety and non-safety applications. These applications aim at improving road safety traffic and assisting drivers. For example, they may warn of dangerous situations and accidents that may occur on the roads and provide internet access and infotainment services. To provide the transfer of information between a group of vehicles within range of each other or between vehicles and the infrastructure, VANETs allow for Vehicle-to-Vehicle (V2V) and Vehicle-to-Infrastructure (V2I) communications $[2,3]$. 
Safety applications impose stringent requirements in terms of Quality of Service (QoS), given the need to achieve bounded delays and reliable communications. Guaranteeing high QoS is an major challenge in VANETs due to the high mobility of nodes, frequent changes in network topology and lack of a central control. In order to satisfy these requirements, therefore, it is necessary to have a QoS model provisioning. Moreover, different layers should cooperate to make correct routing decisions. An efficient Medium Access Control (MAC) is required to provide a broadcast service that respects the necessary requirements. Contentionfree MAC protocols based on the Time Division Multiple Access (TDMA) technique provide considerable benefits, such as collision prevention [4]. Since central coordination is absent in a VANET topology, the propagation of a safety messages to the set of vehicles must be relayed through multiple relay vehicles. Furthermore, building a new multi-hop broadcast scheme of emergency messages seems difficult due to the very nature of VANETs. Many MAC-aware routing and broadcasting protocols have been designed in order to provide multihop communications and disseminate safety messages in a timely manner. In this paper, we present a cross-layer design for multi-hop broadcast of emergency warning messages called AS-DTMAC Multihop. Our approach mixes MAC and routing layers together for an efficient dissemination by selecting the best relay based on the AS-DTMAC [5] [6] protocol, which uses an active signaling technique to eliminate the problem of access collisions during the slot assignment process. In AS-DTMAC Multihop, we adjusted the active signaling process to let the furthest vehicle acquire the dedicated forwarding slot and thus to speed up the dissemination of warning messages.

The remainder of this paper is organized in 4 sections. The next section discusses relevant related work. In Section 3, we present our message broadcast cross-layer solution by presenting first the proposed MAC strategy and then the forwarding strategy. In Section 4, we evaluate the performance of our protocol by presenting simulations results. Finally, in Section 5 , we conclude the paper and outline future work.

\section{Related work}

Recently, several MAC-aware routing protocols have been proposed in the literature to efficiently support multi-hop communication and disseminate safety messages in vehicular networks in a timely manner. These proposals make use of different parameters from the MAC layer, like transmission time slot allocation, channel state, and collision probability, to improve data dissemination in VANETs. A detailed review of these cross-layer routing approaches for VANET is given in [25]. In this section, we focus on solutions dedicated to multi-hop emergency messages broadcast.

One of the earliest approaches to disseminating broadcast packets is called OB-VAN [22]. It is an opportunistic routing protocol that uses a modified 802.11 MAC layer. OB-VAN uses an acknowledgement scheme to choose relay nodes. Choosing the best relay node is performed by using an active signaling technique. Nodes that have captured the packet, transmit a short acknowledgement made up of signaling bursts, calculated based on the distance criterion just after receiving the packet. This scheme is a generalized CSMA/CA where the backoff technique is replaced by the active signaling technique. To prohibit interference on signaling bursts, OB-VAN uses the CDMA spreading code. Signaling bursts can be presented by 0 or 1. 0 denotes a listening interval and 1 denotes a transmission interval. This binary sequence is composed of two parts. The first part, is dedicated to optimizing the criterion for the best relay selection while the second is used to discriminate between nodes and permit the winner 
to relay the data packet.

Another TDMA-based routing protocol designed for warning message dissemination on bi-directional highways is proposed in [23]. This approach, called Priority-Based DirectionAware Media Access Control (PDMAC), classifies nodes as either cluster heads (CHs) or ordinary vehicles (OVs).

To disseminate warning messages, PDMAC develops a three-tier priority assignment process. The first tier is Direction-Based Relay Selection. A source disseminates to its neighbors a request message (REQ) that indicates its direction, destination, etc. and reserves all available time slots in this frame for itself. Neighbors respond with an acknowledgment message (ACK) that contains all free time slots and the slot to be assigned for the transmission of the message according to the severity level of the message. The node selected as the best relay is the one that is closest in distance to the destination and is moving in the direction towards it. The second tier is the Priority on the Basis of Message Type. PDMAC prioritizes warning messages over non warning messages by adding a bit in the message header to indicate the type of the message. Finally, the third tier is Priority on the Basis of Severity Levels to differentiate between different warning messages depending on their severity levels by computing the collision probability. In this case, warning messages are classified into 3 levels. In the case of a lowest priority message, the sender should wait for a free time slot to send. If it is a second level priority, it requests the release of a slot of another non-warning or warning message with lower priority. Otherwise, in the case of a highest priority level message, it is mandatory to release on the time slot of a non-warning or a lower-priority message.

A recent protocol called Multi-Channel Token Ring Protocol (MCTRP) is presented in [24]. MCTRP employs the multi-channel structure defined in IEEE 802.11p. The network is composed of multiple virtual rings. Nodes are classified into 5 types: Ring Founder Node (RFN), Token Holder Node (THN), Ring Member Node (RMN), Dissociative Node (DN), and Semi-Dissociative Node (SDN). There are 2 types of radio: Radio-I and Radio-II. A DN uses only Radio-I since it does not belong to any ring, but the other nodes use both of them. Also, the time system is partitioned into a control period and a data period.

The MCTRP protocol follows 3 sub-protocols. The first sub-protocol is the Ring Coordination Protocol, which manages rings and nodes and schedules Service CHannels $(\mathrm{SCH})$ for each ring. First, the Ring Initialization Process consists of sending a Ring Founding Message (RFM) that includes a selected SCH number for intra-ring data communications and waiting for an invitation. After establishing a ring, a Joining Invitation Message (JIM), which includes some information such as the SCH number, the speed, etc., will be broadcasted by the RFN to the DNs. The DN will reply to the RFN with a Joining Acknowledgement Message (JAM) if the difference between its moving speed and that of the RFN is smaller than a predefined speed threshold. Other messages will be exchanged between RFN, DN and RMN such as Connection Notification Messages (CNMs), Connecting Successor Messages (CSMs), etc. using the contention-based CSMA/CA scheme. The second sub-protocol is the Emergency message exchange protocol. To efficiently deliver emergency messages, MCTRP uses Radio-I or Radio-II, depending on the case. This can be done through 4 steps. Firstly, when an RMN detects an accident, it sends an emergency message to its RFN by adopting CSMA/CA and using Radio-II. Secondly, the RFN node replies with an acknowledgement to the RMN, and then broadcasts the emergency message to all its RMNs using Radio-II. Thirdly, it also broadcasts the message to its neighboring DNs, SDNs, RFNs using Radio-I. Finally, neighboring RFNs rebroadcast the emergency message again to their RMNs using Radio-II. 
The third sub-protocol is the Data Exchange Protocol. Two types of data communications exist: inter-ring data communications where packets are transmitted using CSMA/CA and intra-ring data communications where data packets are transmitted using a token based mechanism. After receiving a token, a node can transmit data during a token holding time and then pass the token to its successor.

\section{Cross-Layer Solution for Emergency Messages Multi-hop Dissemination}

Cross-Layer design is an emerging proposal to support flexible layer approaches in VANETs [25]. As described above, the recent ongoing research has shown increased interest in protocols that rely on interactions between different layers. In this paper, we propose to exploit the relation between MAC and network layers, in an effort to improve the performance of the Multi-hop Broadcast of DENM in Vehicular Networks. We propose the idea of combining an approach based on a MAC layer protocol named AS-DTMAC with a forwarding strategy. Figure 1 represents the general architecture of our message broadcast cross-layer solution. In this section, we first focus on the MAC protocol principle and then we describe the forwarding strategy adopted.

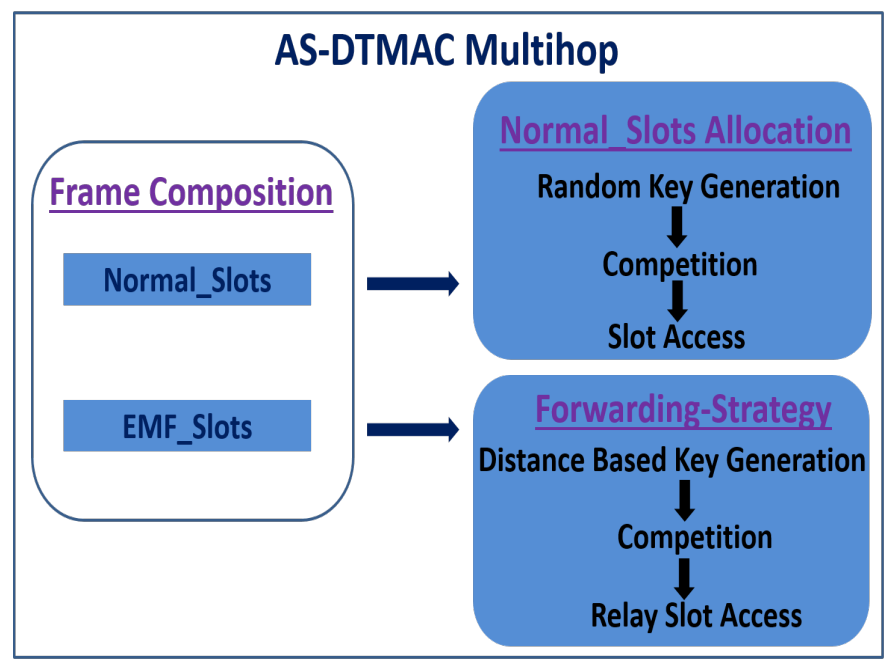

Fig. 1. AS-DTMAC Multihop Architecture 


\subsection{Mac Strategy}

Time Division Multiple Access (TDMA) is a contention-free MAC $^{6}$ protocol with scheduled channel. It is mostly used for safety applications in order to satisfy real-time constraints. Nevertheless, while using TDMA with distributed schemes, the access collision ${ }^{7}$ problem can occur (see [2] for more details). Very many protocols are susceptible to this problem, including: ADHOC MAC [15], VeMAC [18], DTMAC [4], R-ALOHA [17]. To solve this problem, we have designed in [5] [8] [9] [10], an efficient mechanism named active signaling and we apply it in the DTMAC's random slot selection process.

As shown in Figure 2, frame composition in AS-DTMAC Multihop is slightly different from that of AS-DTMAC [5]. In AS-DTMAC Multihop, each frame is composed of 100 slots and it is divided into three sets denoted as $S 0, S 1$ and $S 2$, corresponding to three successive zones. AS-DTMAC Multihop defines two types of slots: Normal_Slot and Emergency_Message_Forwarding_Slot or EMF_Slot. Normal_slots are used by each vehicle to send data, as in AS-DTMAC. However, EMF_Slots are special slots dedicated, by ASDTMAC Multihop, to forwarding emergency messages. In each frame, three slots, namely the first slot of each set $S_{i}$ (i.e. Slot $0, S_{0} \operatorname{sot}_{34}$ and $S_{\text {Sot }} 67$ ), are defined as EMF_Slots. This choice can be explained by the fact that emergency messages are time-sensitive, hence choosing the first slot in each set to forward them will speed up warning message dissemination.

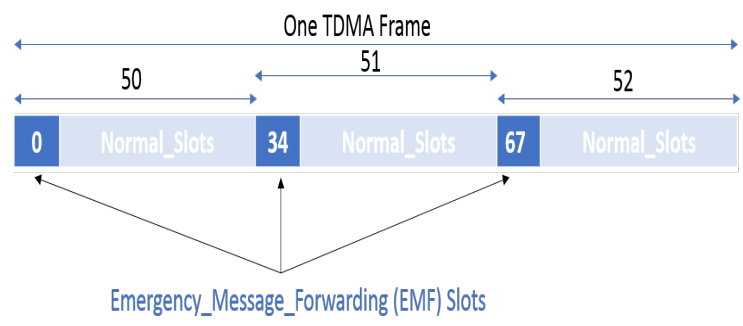

Fig. 2. AS-DTMAC Multihop Frame

As illustrated in Figure 3, whether normal or EMF, a slot is usually formed by two time intervals. The first one, is dedicated to the selection process. However, the second is held by the winner of the competition to transmit its payload packet. In the first interval, a random binary key is generated by every node to compete for a slot. Hopefully, at the end of the time interval, only one node will remain as the winner. The key is a succession of bits $(0,1)$. ' 1 ' means that the vehicle with a packet to send will transmits during the signaling bursts. ' 0 ' means that the vehicle with a packet to send senses the channel during this mini-slot. When a vehicle selects a listening period and senses a transmission, the competition to get the slot

\footnotetext{
${ }^{6}$ According to the control scheme used to access the channel, MAC random access protocols are categorized into: contention-based or contention-free [2].

${ }^{7}$ The access collision problem is can occur when using distributed schemes. It happens when two or more vehicles within the same two-hop neighborhood attempt to access the same available time slot [2].
} 
is over. For instance, a vehicle that draws the key '01001110' will listen during the first minislot and if no competing transmission is sensed during this mini-slot, it will transmit during the next mini-slot. The following two steps of the selection process in our example, will be two listening periods. The selection process continues using the same rule until the key is completely used up.

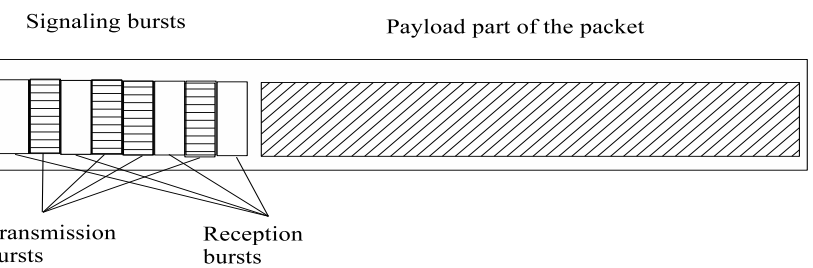

Fig. 3. Structure of the AS-DTMAC slot

\subsection{Forwarding Strategy}

Given the high sensitivity of emergency messages to delay constraints and collisions, it is crucial to propose an efficient multi-hop dissemination scheme that avoids these problems. Since the active signaling technique solves the collision problem, our forwarding strategy is based on an adapted version of the Active Signaling-DTMAC protocol.

As shown in Figure 4, when a source node, $S_{N}$, detects a warning event it has to broadcast an emergency message. Vehicle $S_{N}$ sends the message during its reserved Normal_Slot. All nodes that receive the message and that are located behind the sender: situated in the sender's zone or in the adjacent one (in the sender's transmission range), will compete to forward the received emergency message to other vehicles during the EMF_Slot. To avoid access collision on this slot, each vehicle generates a binary key based on the distance that separates it from the sender. The key is composed of mini-slots. As explained in the previous section, these mini-slots take the value ' 1 ' or ' 0 '. ' 1 ' means that the node is in a transmission mode and ' 0 ' means that the node is in a listening mode. The forwarding strategy consists of selecting the best next relay from the list of vehicles that have generated keys. The winner node will forward the message to the rest of the nodes situated in the opposite direction of the sender vehicle in order to propagate the message as far as possible.

In practice, the winner is always the furthest vehicle from the transmitter. This is counted as a benefit in terms of packet propagation since the emergency message will be quickly broadcasted and the danger will be avoided.

The transmitter sends a message in its own slot reserved in the set dedicated for the zone to which it belongs. So, the forwarding will take place in the first slot of the next set.

To give a clearer idea of our forwarding strategy, we consider the example shown in Figure 5. In this example, the sender, which is the red vehicle, sends an emergency message during its slot (slot 8). In this case, the competition and the first relay of the message will take place in the slot 34. The black vehicle (which is the furthest vehicle) is the winner of the competition and it will forward the message to the other nodes. The same process will be repeated in the next hop until all vehicles have been informed of the potentially dangerous situation. 


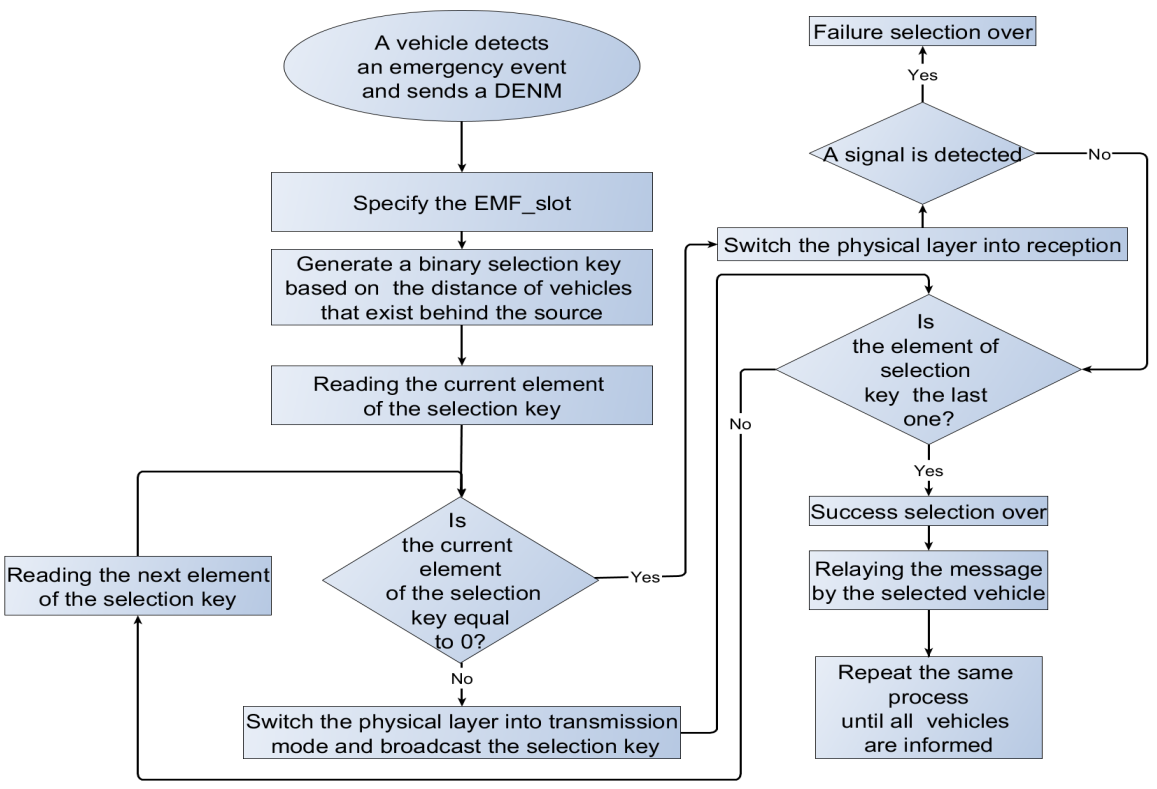

Fig. 4. Forwarding Strategy Flowchart.

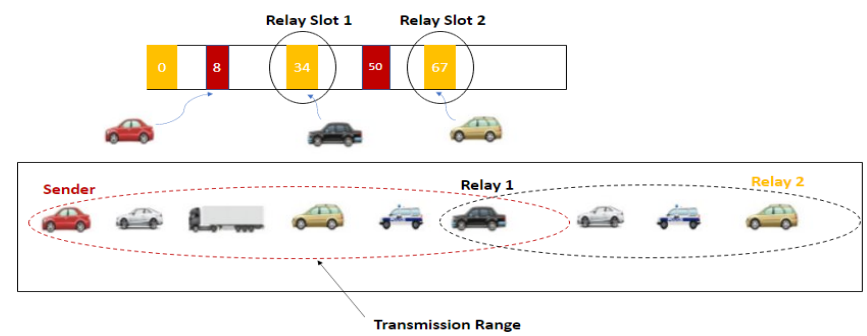

Fig. 5. Forwarding Strategy

\section{Performance evaluation)}

- Latency: this defines the time between the first broadcast of the message and the time of its reception by the last vehicle on the road.

- Packet Loss: as shown below in the equation, the packet loss percentage defines the number of lost packets that are not received, divided by the total number of packets that should be received. The formula to compute the packet loss is defined as follows:

Packet Loss $(\%)=\frac{\text { Losses Packets }}{\text { Total Number of Packets }}$ 
- Number of Forwarders: this metric defines the number of forwarders (relays) needed to relay a message.

- Used Bandwidth: the used bandwidth metric represents the total number of packets received by vehicles.

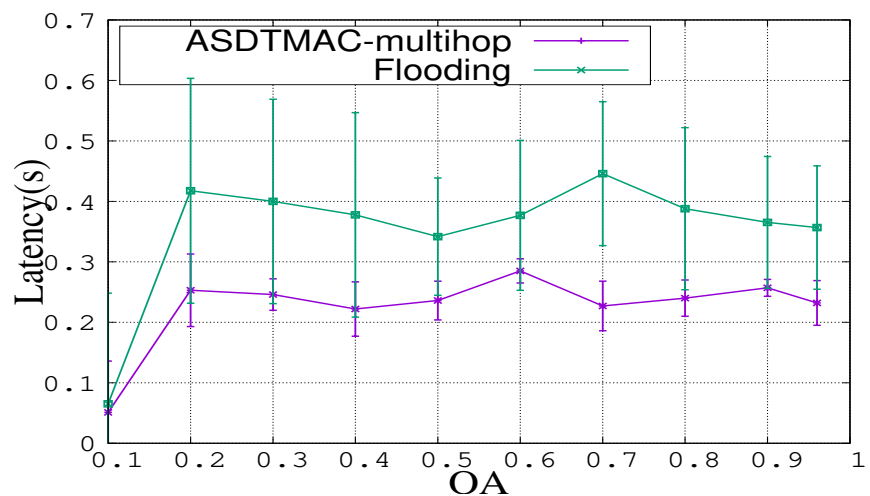

Fig. 6. Latency versus channel occupancy for AS-DTMAC Multi-hop and Flooding with $S S D=20$ and error bar (95\% confidence interval).

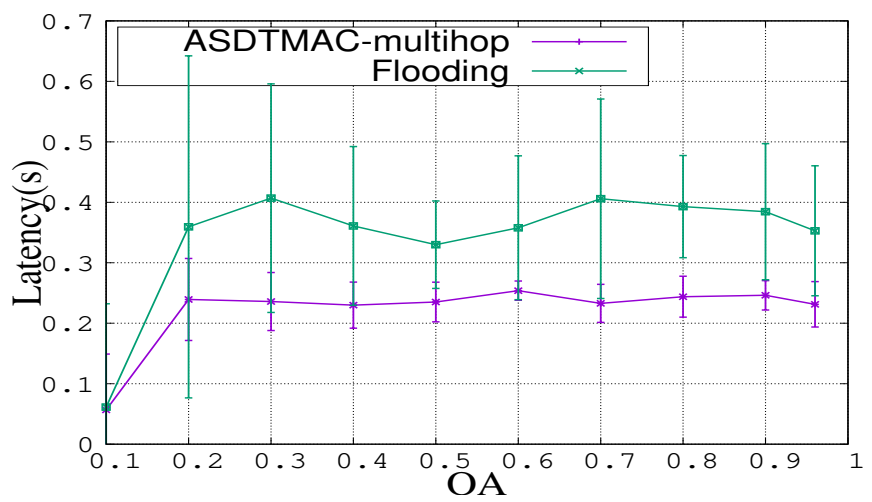

Fig. 7. Latency versus channel occupancy for AS-DTMAC Multi-hop and Flooding with $S S D=30$ and error bar (95\% confidence interval).

Now, We move on to the Packet Loss metric. Figure 8 presents the packet loss versus OA in percent. The error bars are for a $95 \%$ confidence interval. It is clear that AS-DTMAC Multihop has $0 \%$ of packet losses, whereas in Flooding we find a considerable packet loss rate 
that can reach more than $80 \%$ in high traffic level density conditions. As we have explained above, in the Flooding mechanism, every vehicle that has received the message will attempt to forward it and this will cause a high interference in the Flooding scheme. As a result, many packets will be lost.

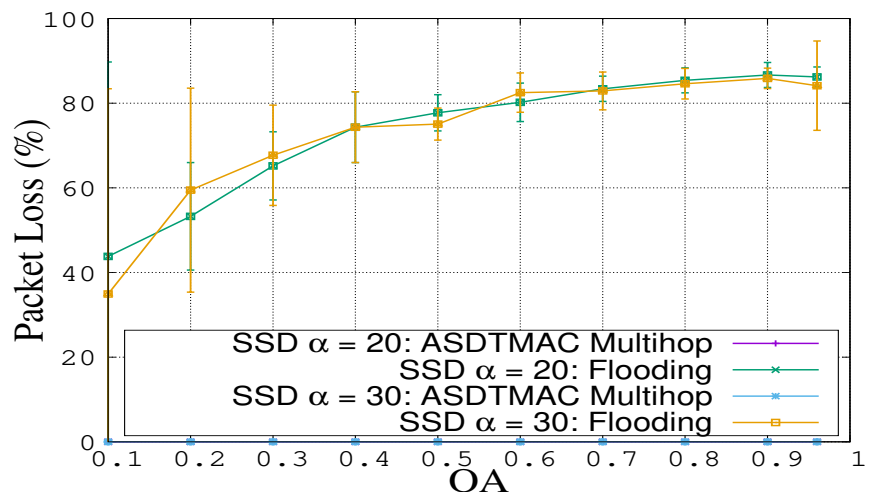

Fig. 8. Packet Loss versus channel occupancy for AS-DTMAC Multi-hop and Flooding with error bar ( $95 \%$ confidence interval).

We now evaluate the Number of Forwarders metric. In Figure 9 and Figure 10, we show the number of forwarders needed for each approach (AS-DTMAC Multihop and flooding) to warn of an emergency event versus OA. Figure 9 represents results with a $S S D$ equal to $20(\mathrm{~km} / \mathrm{h})$ while Figure 10 with a $S S D$ equal to $30(\mathrm{~km} / \mathrm{h})$. As we can see, AS-DTMAC Multihop requires fewer resources, this will reduce the channel occupancy. In contrast, the flooding technique uses more resources because all the vehicles that received the packet will attempt to forward it.

We move on to the Used Bandwidth metric results. In Figures 11 and 12, we plot the used bandwidth versus OA for both AS-DTMAC Multihop and flooding. This metric, represents the total number of received packets. In flooding, we notice that it provides a high value compared to AS-DTMAC Multihop. In our approach, at every hop, only one winner vehicle will relay the packet to its neighbors, whereas in flooding every receiver will relay the packet and thus vehicles could receive the packet several times.

Finally, we compare the dissemination delay achieved by AS-DTMAC Multihop and Flooding to the estimated delay. We begin by deriving an analytic expression of the estimated delay. As illustrated in Figure 13, to deliver an emergency message from $V_{1}$ to $V_{4}$, one frame is sufficient. In fact, the message is relayed 3 times $\left(n_{0}+n_{1}+n_{2}=\tau\right.$ slots $=$ one frame $)$. Based on this information, we derived an analytical formula to compute the estimated delay needed to deliver a message from a source $i$ to a vehicle $j$ separated by such a distance. As defined in [7], the ED is defined in the equation as following:

$$
E D=\frac{D i s t_{i, j}}{3 * R} * \tau * s_{d}
$$




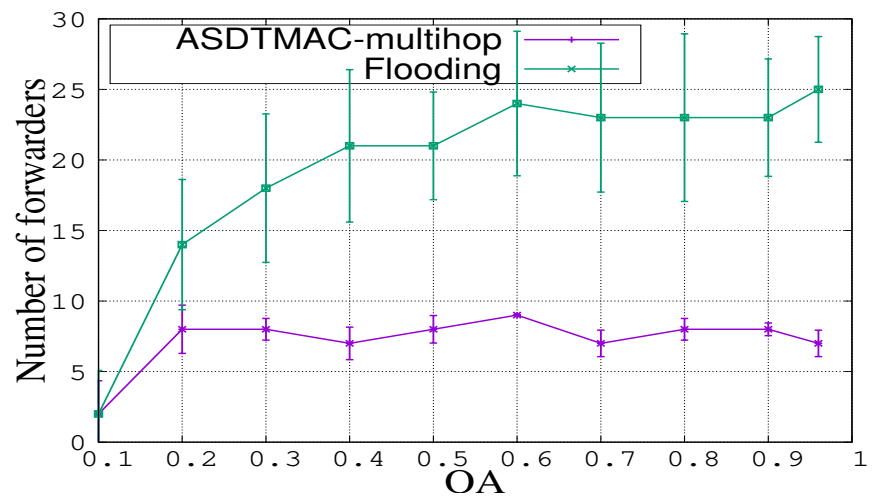

Fig. 9. Number of forwarders versus channel occupancy for AS-DTMAC Multi-hop and Flooding with $S S D=20$ and error bar (95\% confidence interval).

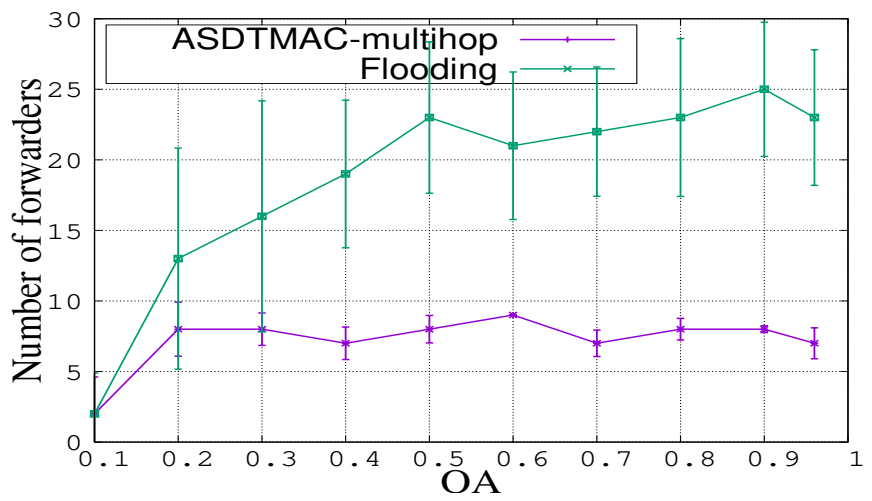

Fig. 10. Number of forwarders versus channel occupancy for AS-DTMAC Multi-hop and Flooding with $S S D=30$ and error bar (95\% confidence interval).

Where $i, j, R, \tau$, Dist $_{i, j}$ and $s_{d}$ are respectively the sender, the receiver, the transmission range, the length of the frame, the distance between the sender and the receiver and the duration of the slot.

Figure 14 presents the estimated delay to propagate a packet from a source to a receiver versus the distance between them.

We can observe from this figure that AS-DTMAC Multihop can provide a shorter delay than the estimated one. This can be explained by the fact that the forwarder will send in one of the reserved slots of forwarding (Slot or Slot $_{34}$ or Slot $_{67}$ ). The delay will depend on the vehicle's position (following the AS-DMAC Multihop scheme: vehicles can access only the set of slot of their area): they can reserve at the beginning, at the middle or at the end. However, due to the interference in the flooding approach, the forwarding operation may not occur in 


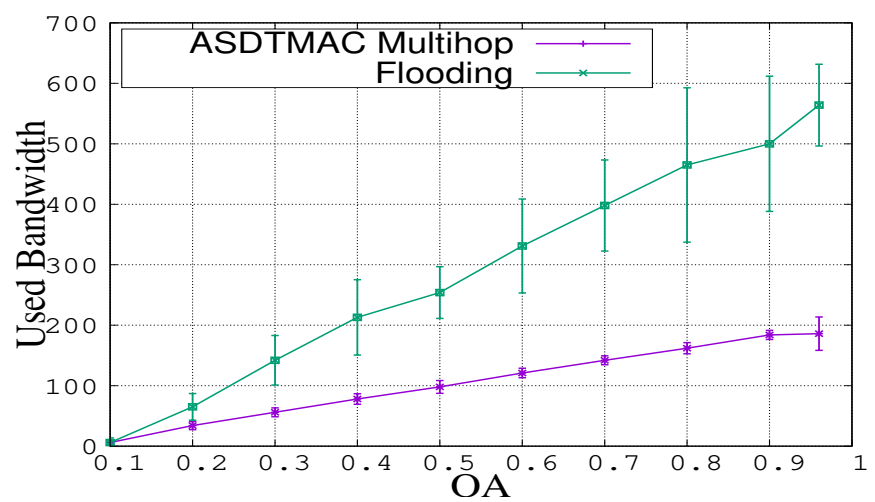

Fig. 11. Used Bandwidth versus channel occupancy for AS-DTMAC Multi-hop and Flooding with $S S D=20$ and error bar (95\% confidence interval).

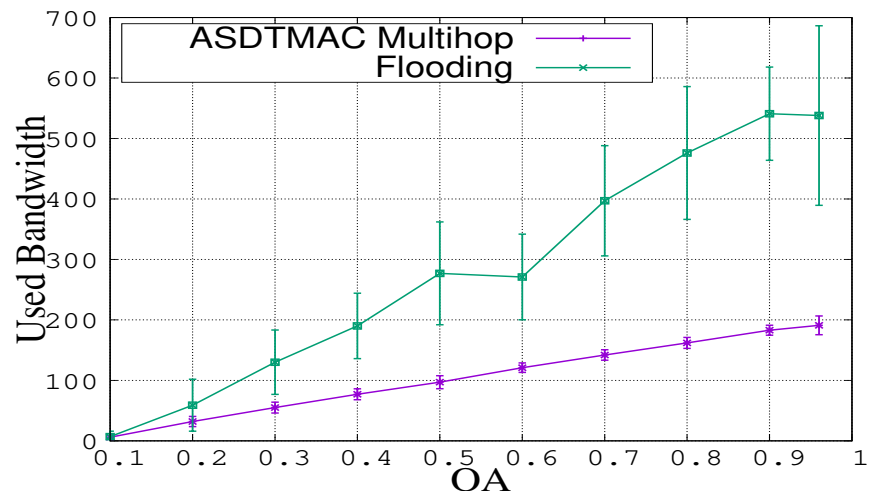

Fig. 12. Used Bandwidth versus channel occupancy for AS-DTMAC Multi-hop and Flooding with $S S D=30$ and error bar (95\% confidence interval).

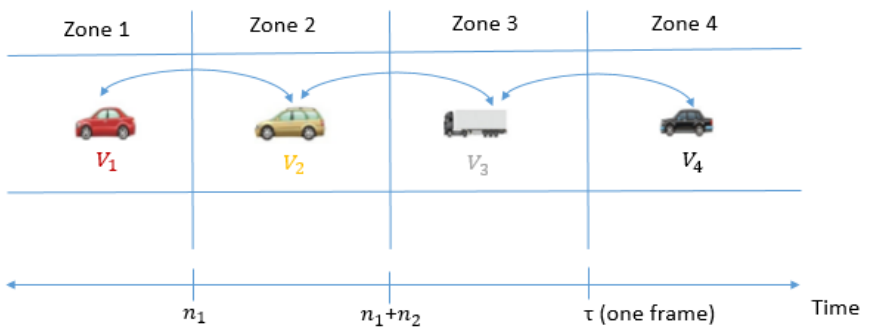

Fig. 13. Message propagation based on TDMA slot information. 
the same frame. If this happens, a vehicle will relay on the next frame. This hypothesis can explain the results obtained.

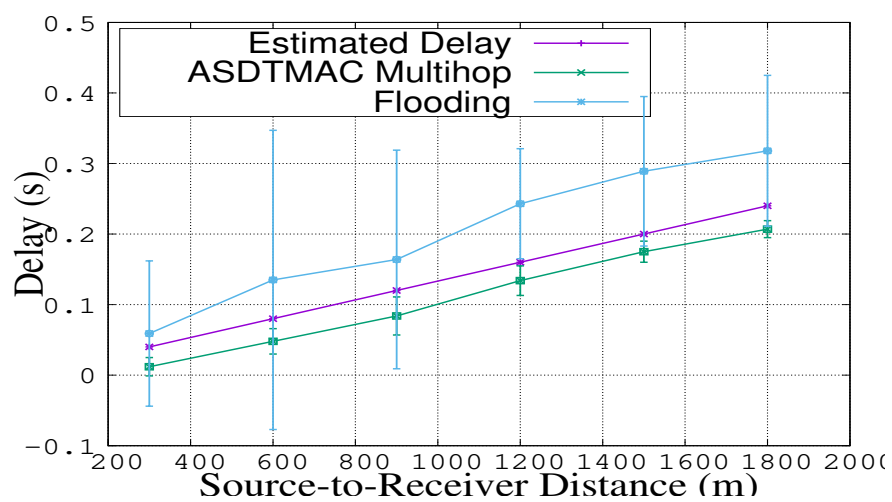

Fig. 14. Estimated Delay versus distance for AS-DTMAC Multi-hop and Flooding with $S S D=30$ and error bar (95\% confidence interval).

\section{Conclusion}

In this paper, we have proposed to enhance DTMAC protocol by integrating active signaling. The simulation results show that AS-DTMAC drastically reduces the access collision rate and allocates slots to all the vehicles in the network in half the time it takes DTMAC to do so. We also presented a use case in the $\mathrm{V} 2 \mathrm{~V}$ for urgent and high priority traffic message like DENM, that can help to avoid an accident, all these new features are very important for the future technology described in the beginning of this paper. As future work, we will do additional simulations to compare with the standard used in V2V (IEEE 802.11p) and we plan to develop an analytical model for AS-DTMAC as well as to investigate further advanced access features that could be provided using the active signaling scheme.

\section{References}

1. Fei Hu, Security and Privacy in Internet of Things (IoTs): Models, Algorithms, and Implementations, ISBN 978-1-4987-2318-3.

2. N. Boulila, M. Hadded, A. Laouiti, and L. A. Saidane, QCH-MAC: A Qos-aware Centralized Hybrid MAC protocol for Vehicular Ad Hoc NETworks, AINA'2018, pp. 55-62, May. 2018.

3. A. Laouiti, A. Qayyum and M-N-M. Saad, Vehicular Ad-Hoc Networks for Smart Cities, In Proceedings of the Second International Workshop of Advances in Intelligent Systems and Computing, Vol. 548, Springer, 2017. 
4. M. Hadded, A. Laouiti, P. Muhlethaler and L. A. Saidane, An infrastructure-free slot assignment algorithm for reliable broadcast of periodic messages in vehicular ad hoc networks, in Vehicular Technology Conference VTC-Fall, Montreal, Canada, Sep. 2016.

5. F. Boukhalfa, M. Hadded, P. Muhlethaler and O. Shagdar, An active signaling mechanism to reduce access collisions in a distributed TDMA based MAC protocol for vehicular networks, Advanced Information Networking and Applications, AINA-2019, Kunibiki Messe, Matsue, Japan March 27 to March 29, 2019

6. F. Boukhalfa, M. Hadded, P. Muhlethaler and O. Shagdar, An Analytical Model for Performance Analysis of An active Signaling-based MAC Protocol for Vehicular Networks, in Vehicular Technology Conference VTC-FALL, Honolulu, Hawaii, USA, Sept. 2019.

7. M. Hadded, P. Muhlethaler, and A. Laouiti, Performance evaluation of a TDMA-based multi-hop communication scheme for reliable delivery of warning messages in vehicular networks, in Proc. Wireless Commun. Mobile Comput. Conf. (IWCMC), 2017, pp. 10291034.

8. F. Boukhalfa, C. Adjih, P. Muhlethaler, M. Hadded and O. Shagdar, Physical and MAC Layer Design for Active Signaling Schemes in Vehicular Networks, WiMob, Thessaloniki, Greece, pp. 86-92, 2020.

9. F. Boukhalfa, M. Hadded, P. Muhlethaler and O. Shagdar, Coexistence of IEEE 802.11p and the TDMA-based AS-DTMAC Protocol,SoftCOM, Split, Croatia, pp. 1-6, 2020.

10. F. Boukhalfa, M. Hadded, P. Muhlethaler and O. Shagdar, Performance evaluation of an active signaling based time-slot scheduling scheme for connected vehicles, Annals of Telecommunications, pp. 1-12, 2020.

11. J. Abate and W. Whitt, Numerical Inversion of Laplace Transforms of Probability Distributions, ORSA Journal on Computing, Vol 7, no 1, pp 38-43, year 1995

12. F. Ye, R. Yim, J. Zhang, and S. Roy, Congestion control to achieve optimal broadcast efficiency in vanets, in IEEE International Conference on Communications (ICC), Cape Town, South Africa, May 2010, pp. 1-5.

13. 802.11p-2010, IEEE standard for information technology - Telecommunications and information exchange between systems - local and metropolitan area networks - specific requirements part 11 : Wireless LAN medium access control (MAC) and physical layer (PHY) and physical layer (PHY) specifications amendment 6 : Wireless access in vehicular environments Std., 2010.

14. R. Uzcategui and G. Acosta-Marum, Wave: A tutorial, IEEE Communications Magazine, vol. 47, no. 5, pp. 126-133, May 2009.

15. F. Borgonovo, A. Capone, M. Cesana, and L. Fratta, Adhoc mac: new mac architecture for ad hoc networks providing efficient and reliable point-to-point and broadcast services, Wireless Networks, vol. 10, no. 4, pp. 359-366, 2004.

16. G. Naik,B. Choudhury and J. M Park, IEEE 802.11 bd \& $5 G$ NR V2X: Evolution of Radio Access Technologies for V2X Communications, IEEE Access journal, vol. 7, pp. 70169-70184, May. 2019.

17. F. Borgonovo, A. Capone, M. Cesana, and L. Fratta, Rr-aloha, a reliable r-aloha broadcast channel for ad-hoc intervehicle communication networks, in IEEE IFIP Annual Mediterranean Ad Hoc Networking Workshop (Med-Hoc-Net), Baia Chia, Italy, 2002.

18. W. Zhuang, H. A. Omar, and L. Lio, Vemac: A novel multichannel mac protocol for vehicular ad hoc networks, in IEEE Conference on Computer Communications Workshops (INFOCOM WKSHPS), Shanghai, China, Aug. 2011, pp. 413-418.

19. Y. Weidong, L. Pan, L. Yan, and Z. Hongsong, Adaptive TDMA slot assignment protocol for vehicular ad-hoc networks, Journal of China Universities of Posts and Telecommunications, vol. 20, no. 1, pp. 11-18, Feb. 2013. 
20. W. Ke, Y. Weidong, L. Pan, and Z. Hongsong, A decentralized adaptive TDMA scheduling strategy for VANETs, in IEEE Wireless Communications and Networking Conference Workshops (WCNCW), Shanghai, China, Apr. 2013, pp. 216-221.

21. F. Karnadi, Z. Mo, and K. chan Lan, Rapid generation of realistic mobility models for VANET, in IEEE WCNC, Hong Kong, China, Mar. 2007, pp. 2506-2511.

22. B. Blaszczyszyn, A. Laouiti, P. Muhlethaler, and Y. Toor, Opportunistic broadcast in VANETs (OB-VAN) using active signaling for relays selection, IEEE International Conference on ITS Telecommunications (ITST'08), pp. 384-389, Oct. 2008.

23. G. Abbas, Z. H. Abbas, S. Haider, T. Baker, S. Boudjit and F. Muhammad, PDMAC: A priority-based enhanced TDMA protocol for warning message dissemination in VANETs, Sensors, vol. 20, pp. 45, 2020.

24. Y. Bi, K.H. Liu, L.X. Cai, and X. Shen, A Multi-Channel Token Ring Protocol for QoS Provisioning in Inter-Vehicle Communications, IEEE Transactions on Wireless Communications, vol. 8, no. 11, pp. 5621-5631, Nov. 2009

25. A. Rebei, M. Hadded, H. Touati, F. Boukhalfa and P. Muhlethaler, "MAC-aware Routing Protocols for Vehicular Ad Hoc Networks: A Survey," 2020 International Conference on Software, Telecommunications and Computer Networks (SoftCOM), Split, Hvar, Croatia, 2020, pp. 1-6, doi: 10.23919/SoftCOM50211.2020.9238249. 